\title{
Comparative study of glucometer and laboratory glucose oxidase method for the estimation of blood glucose levels in neonates
}

\author{
Naaz F. ${ }^{1}$, Gulati R.K. ${ }^{2}$ \\ ${ }^{1}$ Dr. Farah Naaz, Paediatrics, ${ }^{2}$ Dr. R.K.Gulati, Ex-Senior Professor and HOD, both authors are attached with Department \\ of Paediatrics, Government Medical College Kota, Rajasthan, India.
}

Corresponding Author: Dr. Farah Naaz, Paediatrics, Department of Paediatrics, Government Medical College Kota. Address- B 201 Golden Height Apartment, Flat No 502 Rajendra Marg, Bapunagar, Jaipur (Rajasthan) India. E-mail: nfarah30@gmail.com

\begin{abstract}
Introduction: Hypoglycemia is one of the most common metabolic problems encountered in neonates. The glucose oxidase method used in the laboratory for determining the blood glucose concentration is precise and specific for glucose. The glucometers are often used for blood glucose estimation in NICU. Objectives: This is a prospective study done to determine the efficacy of glucometer in estimation of blood glucose levels in neonates in comparison with the laboratory values. Methods: 170 neonates admitted in NICU in Jay Kay Lon Hospital, Kota associated with Government Medical College Kota with varied symptomatology were enrolled in this study. Blood glucose estimation was done by glucometer and laboratory method using the same venous sample at the time of admission. Statistical analysis was done by using Pearson correlation. Hypoglycemia was defined as blood glucose level $<45 \mathrm{mg} \%$. Laboratory value was taken as gold standard. Results: Hypoglycemia was common in neonates with risk factors like prematurity, meconium aspiration, septicemia, birth asphyxia etc. In our study Glucometer had a specificity of $98.20 \%$, sensitivity of $64.48 \%$, positive predictive value of $95 \%$, negative predictive value of $83.84 \%$ and accuracy of $86.47 \%$. There was good correlation and no significant difference between two methods in the range between $45 \mathrm{mg} / \mathrm{dl}$ and $145 \mathrm{mg} / \mathrm{dl}$ but not at very high or very low glucose concentrations Conclusion: The 'Point of care "device - glucometer as a sole measuring device to screen neonatal hypoglycemia is not satisfactory and confirmation with the laboratory measurements of plasma glucose is still of up most importance.
\end{abstract}

Keywords: Hypoglycemia, Neonates, Glucometer, Glucose oxidase method

\section{Introduction}

Alteration in blood glucose levels in newborns is difficult to detect clinically. Hence a reliable "point of care"device (glucometer) for early detection and treatment is needed. Hypoglycemia is one of the most common metabolic problems encountered in the newborns. The overall incidence of hypoglycemia in neonates varies from 0.2 to $11.4 \%[1,2]$. However in the presence of certain risk factors i.e. small for date, large for date, infants of diabetic mothers, prematurity etc., the probability of hypoglycemia increases many folds [1].

Hypoglycemia in neonates can be symptomatic and asymptomatic $[3,4,5]$. The most common symptoms such as jitteriness, convulsions, apathy, hypotonia,

\footnotetext{
Manuscript received: $18^{\text {th }}$ February 2019

Reviewed: $28^{\text {th }}$ February 2019

Author Corrected: $8^{\text {th }}$ March 2019

Accepted for Publication: 14 $4^{\text {th }}$ March 2019
}

coma, refusal to feed, cyanosis, high pitched cry, hypothermia are very nonspecific and especially in small sick infants, these symptoms may be easily missed. Therefore, hypoglycemia must always be confirmed biochemically and by response to treatment. Hypoglycemia is known to be associated with brain dysfunction and neuromotor developmental retardation in both symptomatic and asymptomatic cases $[1,6]$.

In symptomatic infant, plasma glucose concentration should be measured and if the value is $<45 \mathrm{mg} \%$, clinical interventions aimed at increasing blood glucose concentrations are indicated [7].

The methods for determining blood/plasma glucose concentration include reductiometric method, glucose oxidase method and hexokinase method $[1,8]$. The glucose oxidase method used in the laboratory for 


\section{Original Research Article}

determining the blood glucose concentration is precise and specific for glucose [1]. As it is usually performed in the main laboratory, the results are not available quickly enough for timely appropriate management. The glucometers are often used for blood glucose estimation in NICU.

Many studies have shown that their results co-relate well with the laboratory measured glucose levels in the normoglycemic and hyperglycemic range but are not satisfactory in the lower range [9-16]. We did a comparative study of blood glucose level in neonate using glucometer and laboratory glucose oxidase method to determine the efficacy of glucometer in comparison with the laboratory values.

\section{Materials and Methods}

Source of data: The neonates admitted to NICU over a period of 3 months in Jay Kay Lon Hospital, Kota associated with Government Medical College Kota.

Study design: Prospective study

\section{Sample size: 170}

\section{Inclusion criteria}

1.Neonates admitted in Jay Kay Lon Hospital Neonatal Intensive Care Unit (NICU)associated with Government medical college, Kota.

2.Both sex

\section{Exclusion criteria}

1. Infant $>28$ Days

2. Neonates with PCV less than $40 \%$ more than $65 \%$

3. Out born or referral case
Method of collection of data: Collection of data was by relevant investigations i.e. glucose estimation by glucometer and laboratory glucose oxidase method.

Methodology: At admission, a detailed history was taken and a thorough physical examination was performed so as to fulfill the inclusion and exclusion criteria laid down in the study protocol.

Capillary blood glucose levels measured using glucometer (ONE TOUCH-select simple) ${ }^{\text {TM }}$ with plasma obtained from heel prick using a stylet under aseptic precautions. Simultaneously $2 \mathrm{ml}$ of blood drawn from peripheral vein was collected in fluoride tubes and sent to central clinical biochemistry laboratory for plasma glucose measurement using glucose oxidase method.

The analysis was done on emergency basis within half an hour of collection of blood sample. Routine quality control check was followed for the analysis. A calibrated check using the manufacturer supplied control cuvette was performed once a day on the glucose analyser.

Hypoglycemia was defined as blood glucose levels less than $45 \mathrm{mg} / \mathrm{dl}$ Hyperglycemia was defined as blood glucose levels more than $145 \mathrm{mg} / \mathrm{dl}$. Blood glucose levels $45-145 \mathrm{mg} / \mathrm{dl}$ was considered as normal. Laboratory value of glucose was taken as gold standard.

Statistical analysis: Statistical methods used were Descriptive Statistics, Frequencies, Contingency Coefficient Test (Crosstabs) and correlation. All the statistical analysis were carried out through the software SPSS for Windows (version 16.0).

\section{Results}

170 neonates admitted in NICU of JKLON hospital, Kota were enrolled in our study. Out of them 110 were males and 60 were females. 102 were term, 68 were preterm and majority being less than 7 days.

They had varied etiology and clinical diagnosis of birth asphyxia, meconium aspiration syndrome/meconium stained amniotic fluid, neonatal sepsis, neonatal hyperbilirubinemia, respiratory distress syndrome and others (including preterm for nutritional and supportive care, seizures for evaluation, premature rupture of membranes, acute kidney injury, tracheo-esophageal fistula and other congenital malformations).

Neonates were classified according to their gestational age by WHO Fenton charts. Maximum neonates (40.6\%) were appropriate for gestational age (AGA). Remaining $17 \%$ were small for gestational age (SGA) and $2.4 \%$ were large for gestational age (LGA).

Table 1,2 and figure 1 shows that there is a large correlation between the glucometer and lab values when the blood glucose values, as determined by the gold standard is $>45 \mathrm{mg} \%$. But when the lab values (gold standard) are $<45 \mathrm{mg} \%$ then there is just moderate correlation between the two. The accuracy of glucometer to detect hypoglycemia in newborns in comparison with gold standard (lab glucose oxidase method) is $86.47 \%$. 


\section{Original Research Article}

Table-1: Diagnostic value of Glucometer in relation to Lab value in detecting the hypoglycemia.

\begin{tabular}{|c|c|}
\hline Value & Percentage \\
\hline Sensitivity & $64.40 \%$ \\
\hline Specificity & $98.20 \%$ \\
\hline PPV & $95 \%$ \\
\hline NPV & $83.84 \%$ \\
\hline Accuracy & $86.47 \%$ \\
\hline
\end{tabular}

Table-2: Pearson correlation: lab glucose oxidase method and glucometer (capillary sample).

\begin{tabular}{|l|l|c|c|c|c|}
\hline \multicolumn{2}{|c|}{} & \multicolumn{3}{|c|}{ Lab Glucose Oxidase Method (MG/DL) } & \multirow{2}{*}{ Total } \\
\cline { 2 - 6 } \multicolumn{2}{|c|}{} & $<\mathbf{4 5}$ & $\mathbf{4 5 - 1 4 5}$ & $>\mathbf{1 4 5}$ & \\
\hline \multirow{3}{*}{$\begin{array}{l}\text { Glucometer (Capillary Sample) } \\
\text { In MG/DL }\end{array}$} & $<\mathbf{4 5}$ & 38 & 2 & 0 & 40 \\
\cline { 2 - 6 } & $\mathbf{4 5 - 1 4 5}$ & 21 & 100 & 4 & 125 \\
\cline { 2 - 6 } & $>\mathbf{1 4 5}$ & 0 & 1 & 4 & 5 \\
\hline \multicolumn{2}{|l|}{\begin{tabular}{l} 
Total \\
\hline
\end{tabular}}
\end{tabular}

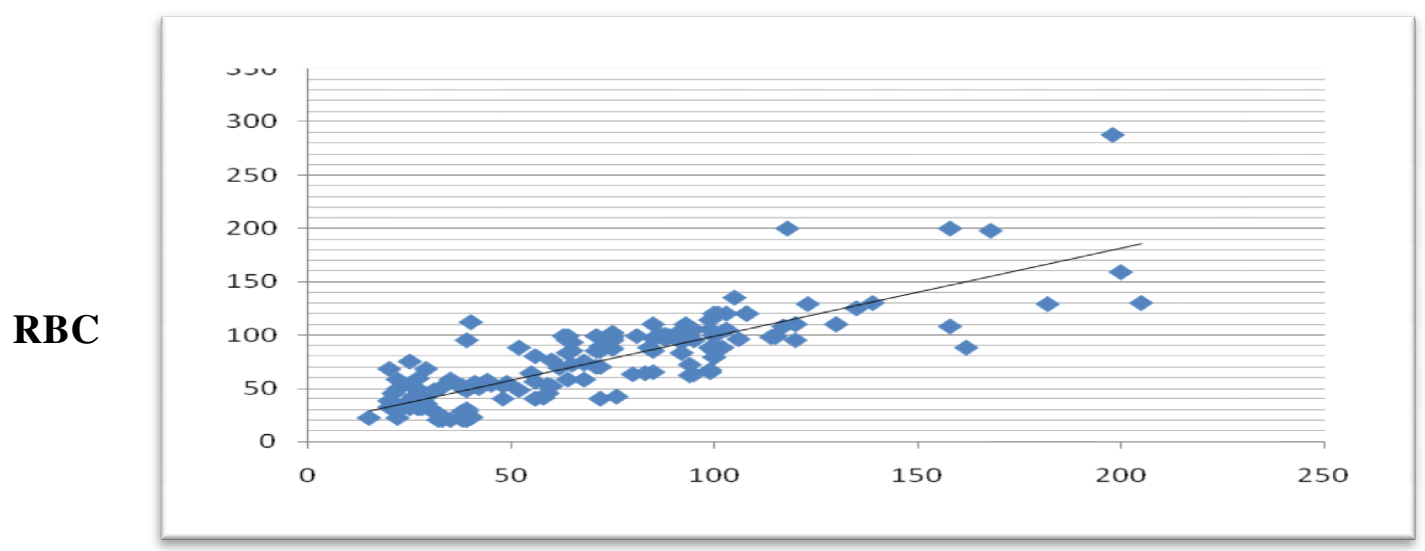

\section{Lab RBS}

Figure-1: Pearson correlation: lab glucose oxidase and glucometer (Capillary)

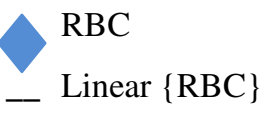

\begin{tabular}{|c|c|}
\hline Contingency coefficient $(\mathbf{r})$ & p value \\
\hline+0.837 & 0.000 \\
\hline
\end{tabular}

\section{Discussion}

Neonatal hypoglycemia is a common metabolic disorder and the operational threshold values of blood glucose less than $45 \mathrm{mg} / \mathrm{dl}$ should be used to guide management.The normal range of blood glucose is variable and depends upon factors like birth-weight, gestational age, body stores, feeding status, availability of energy sources as well as the presence or absence of disease [19,21]. All "at risk" neonates and sick infants should be monitored for blood glucose levels.In our study we evaluated the efficacy of glucometers in estimating the blood glucose levels in newborns, in comparison with laboratory glucose oxidase method. Main stress was laid on detection of hypoglycemia by both the methods and to know if glucometer is a good screening tool to detect hypoglycemia. Laboratory glucose oxidase method of blood glucose estimation was taken as gold standard. Hypoglycemia was defined as blood glucose level less than $45 \mathrm{mg} \%$ as defined by the study by Corn lath et al[19]. Our study showed that One Touch (select simple) glucometer had sensitivity of $64.48 \%$ to detect hypoglycemia and specificity of $98.20 \%$ to detect hypoglycemia which is in correlation with other studies. This 


\section{Original Research Article}

is in accordance with Ngerncham S et al[17] and Hamid MH et al[16] respectively (table 3). It was observed from our study that glucometer had a positive predictive value of $95.00 \%$ which in contrast to Ngerncham S et al[17] and Ho HT et al[14] is low and negative predictive value of $83.84 \%$ which in contrast to Ngerncham S et al[17] and Ho HT et al. is low [14] (table 3). The accuracy of glucometer to detect hypoglycemia in newborns in comparison with gold standard (lab glucose oxidase method) is $86.47 \%$.

Table-3: Comparison of sensitivity, specificity, PPV \& NPV of various studies.

\begin{tabular}{|c|c|c|c|c|}
\hline & Sensitivity & Specificity & Positive predictive value & Negative predictive value \\
\hline Our study & $\mathbf{6 4 . 4 0 \%}$ & $\mathbf{9 8 . 2 0} \%$ & $\mathbf{9 5 . 0 0 \%}$ & $\mathbf{8 3 . 8 4 \%}$ \\
\hline Ngerncham S et al [17] & $62 \%$ & $100 \%$ & $100 \%$ & $85.7 \%$ \\
\hline Ho HT et al [14] & $46.7 \%$ & $79.2 \%$ & $73.7 \%$ & $54.3 \%$ \\
\hline
\end{tabular}

Lab glucose oxidase method is considered as gold standard. Although Glucometers are reported to have several advantages in the analysis of glucose in newborns such as short analysis time, small sample size, quick reading, and can be handled even by unskilled personnel, still it remains as a screening tool. It has been shown that most glucometers are inaccurate at very high or very low glucose concentrations and certain variables like haematocrit, altitude, environmental temperature or humidity and hypoxia may affect the result with bedside testing $[15,20]$

Correlation of glucometer and lab value: In our study there was good correlation between two methods in the range between $45 \mathrm{mg} / \mathrm{dl}$ and $145 \mathrm{mg} / \mathrm{dl}$. It has been shown that most glucometers are inaccurate at very high or very low glucose concentrations and certain variables like haematocrit, altitude, environmental temperature or humidity and hypoxia may affect the result with bedside testing[13,15,17].

In our study glucometer readings were higher in babies having glucose values below $45 \mathrm{mg} / \mathrm{dl}$ in relation to laboratory glucose oxidase method. Glucose values measured using laboratory glucose oxidase method was higher in babies who had glucose levels higher than $145 \mathrm{mg} / \mathrm{dl}$ when compared with glucometer. We observed that few babies were overestimated for hypoglycemia when tested with glucometer. So keeping in view the high specificity of glucometer in our study, we conclude that, if the clinician feels neonate has hypoglycemia, but the glucometer gives normal values, it is better to get the blood sugar repeated by glucose oxidase method before giving any treatment.

Hence our results agree with previous studies which concluded that glucose reagent strips should be considered only as aeasy, rapid, point of care test and not as a diagnostic test, due to their questionable reliability $[13,15,18,22]$. The limitation of our study was firstly the small sample size in the higher glucose range $(>145 \mathrm{mg} / \mathrm{dl})$. Further studies on a larger sample in higher glucose range are needed. Secondly glucose estimation in our study did not specify age in hours at the time of blood glucose estimation. Lastly whole blood glucose level at room temperature in-vitro decline at a rate of $5-7 \mathrm{mg} / \mathrm{dl} /$ hour. The use of inhibitors such as fluoride, in collection tubes are used to avoid this problem. The efficacy is best when prepared freshly. But the fluoride tubes used in our study were pre-prepared commercially available tubes.

\section{Conclusion}

There is a variable detection rate of hypoglycemia by glucometer in the studies mentioned and also in our study. Glucometer had good correlation at glucose levels $45-145 \mathrm{mg} / \mathrm{dl}$, still its values in this study do not have sufficient validity to replace laboratory testing in diagnosing hypoglycemia and hyperglycemia. We conclude that glucometer test was satisfactory as aeasy, rapid, point of care test in diagnosing neonatal hypoglycaemia but it can either over or underestimate glucose results. Although the glucometer had good specificity and negative predictive value, confirmation with laboratory measurements of plasma glucose and clinical assessment of the infant are still of the utmost importance.
What this study adds to our existing knowledge: Glucometers (point of care devices) as a sole measuring device to screen neonatal hypoglycemia is not satisfactory and confirmation with the laboratory measurements of plasma glucose is still of up most importance.

Funding: Nil, Conflict of interest: None initiated, Perission from IRB: Yes

\section{References}

1. Singhal PK, Singh M, Paul VK, et al. Neonatal hypoglycemia--clinical profile and glucose requirements. Indian Pediatr. 1992 Feb;29(2):167-71. 


\section{Original Research Article}

2. Williams AF. Hypoglycaemia of the newborn: a review. Bull World Health Organ. 1997;75(3):261-90.

3. Charles AS \&Eugina KP: Disorders of Carbohydrate Metabolism. In: Avery Disease of the Newborn. Teuscch HW, Ballard RA \& Gleason CA. 8TH ed. Philadelphia: SAUNDERS, 2005; 1410-1420.

4. Kleigman RM: Problems In Metabolic Adaptation: Glucose, Calcium and Magnesium. In: Care Of the High Risk Neonate. Klaus MH \&Faranoff AA. 5th ed. Philadelphia: SAUNDERS, 2000; 304-309.

5. Barbara JS and Robert MK: The Endocrine System. In: Nelson Text Book of Pediatrics. Behrman RE, Kliegman RM, Jenson HB. 17TH ed. Philadelphia: SAUNDERS, 2004; 614-616.

6. Alkalay AL, Flores-Sarnat L, Sarnat HB, et al. Brain imaging findings in neonatal hypoglycemia: case report and review of 23 cases. Clin Pediatr (Phila). 2005 NovDec;44(9):783-90. DOI:10.1177/000992280504400906

7. Cornblath M, Hawdon JM, Williams AF, et al. Controversies regarding definition of neonatal hypoglycemia: suggested operational thresholds. Pediatrics. 2000 May;105(5):1141-5.

8. Gregory A. Threatte, John Bernard henry: Carbohydrates. In: Clinical Diagnosis and management by laboratory methods. JB Henry. 19th ed. Noida: Harcourt Asia PTE Ltd, 1999; 194-207

9. Narayan S, Aggarwal R, Deorari AK, et al. Hypoglycemia in the newborn. Indian J Pediatr. 2001 Oct; 68 (10):963-5.

10. Michel A, Küster H, Krebs A, et al. Evaluation of the Glucometer Elite XL device for screening for neonatal hypoglycaemia. Eur J Pediatr. 2005 Nov; 164 (11): 660-4. Epub 2005 Jul 22. DOI:10.1007/s00431005-1733-9

11. Pamela Kirkham, Andrew Watkins. Comparison of two reflectance photometers in the assessment of neonatal hypoglycemia. Arch Dis Child Fetal Neonatal Ed 1995; 73: F170-73.

12. Dahlberg, Marianne, et al. Comparison of a new instant blood glucose method with conventional laboratory analysis in diagnosis of neonatal hypoglycemia. Pediatr Res 1996; 39(4)307.
13. Mehta, Nilesh M, Whitelaw, Andrew, Stone, Janet. Diagnosis of neonatal hypoglycemia at the cot side. A comparison of precision Q.I.D., Hemocue and Hexokinase Methods. Pediatr Res 1999; 45(94): 211A.

14. Ho HT, Yeung WK, Young BW. Evaluation of "point of care" devices in the measurement of low blood glucose in neonatal practice. Arch Dis Child Fetal Neonatal Ed. 2004 Jul;89(4):F356-9. DOI:10.1136/adc. 2003.033548

15. Ellis M, Manadar DS, Manadar N, JM Land, NPatel, AM de L Costello. Comparision of two cotside methods for detection of hypoglycemia among neonates in Nepal. Arch Dis Child Fetal Neonatal Ed 1996; 75: F122-5.

16. Hamid MH, Chishti AL, Maqbool S. Clinical utility and accuracy of a blood glucose meter for the detection of neonatal hypoglycemia. J Coll Physicians Surg Pak. 2004 Apr; 14(4): 225-8. DOI: 04.2004/JCPSP. 225228

17. Ngerncham $S$, Piriyanimit $S$, Kolatat $T$, et al. Validity of two point of care glucometers in the diagnosis of neonatal hypoglycemia. Indian Pediatr. 2012 Aug;49(8):621-5. Epub 2011 Jan 17.

18. Arun kumar De, Biswas R, Samanta M. Study of blood glucose level in normal and low birth weight newborns and impact of early breast feeding in a tertiary care centre. Ann Nigerian Med 2011;5:53-8..

19. Cornblath M, Ichord R. Hypoglycemia in the neonate. SeminPerinatol. 2000 Apr;24(2):136-49.

20.Kilpatrick ES1, Rumley AG, Myint H, et al. The effect of variations in haematocrit, mean cell volume and red blood cell count on reagent strip tests for glucose. Ann Clin Biochem. 1993 Sep;30 ( Pt 5):485-7. DOI:10.1177/000456329303000513

21. Mitanchez D. Glucose regulation in preterm newborn infants. Horm Res. 2007;68(6):265-71. Epub 2007 Jun 20. DOI:10.1159/000104174

22. Harris DL, Battin MR, Weston PJ, et al. Continuous glucose monitoring in newborn babies at risk of hypoglycemia. J Pediatr. 2010 Aug;157(2):198-202.e1. doi: 10.1016/j.jpeds.2010.02.003. Epub 2010 Mar 24.

\section{How to cite this article?}

Naaz F, Gulati R.K. Comparative study of glucometer and laboratory glucose oxidase method for the estimation of blood glucose levels in neonates. Int J Pediatr Res. 2019;6(03):113-117.doi:10.17511/ijpr.2019.i03.02 